Research Paper

\title{
Geometrical Elucidations and Approximation of some Functional Equations in Numerous Variables
}

\author{
HEMEN DUTTA ${ }^{1, *}$ and B V SENTHIL KUMAR ${ }^{2}$ \\ ${ }^{1}$ Department of Mathematics, Guwahati University, Guwahati 781 014, Assam, India \\ ${ }^{2}$ Department of Mathematics, C. Abdul Hakeem College of Engineering and Technology, Melvisharam \\ 632 509, Tamil Nadu, India
}

(Received on 21 March 2018; Accepted on 15 September 2018)

\begin{abstract}
The objective of this paper is to introduce a reciprocal-quadratic difference functional equation and a reciprocal-quadratic adjoint functional equation in copious variables with their geometrical interpretations in Physics and to determine their fundamental stabilities connected with Ulam stability theory in quasi- $\beta$-normed spaces.
\end{abstract}

Keywords: Rational Function, Reciprocal-Quadratic Function; Functional Equation; Rational Functional Equation; Generalized Ulam-Hyers Stability; Quasi- $\beta$-Normed Space

\section{Introduction}

The basis for the exploration of stability of functional equation is the problem solicited first by (Ulam, 1964). The foremost mathematician responded through a partial answer to the question pleaded by Ulam (Hyers, 1941). The response given by Hyers made a foundation stone in the theory of stability of functional equations. The stability result attained by Hyers is called as Hyers-Ulam stability or $\varepsilon$-stability of functional equation. Later, Hyers' result was further promoted (Aoki, 1950) and modified (Rassias, 1978) (stability involving sum of powers of norms), (Rassias, 1982) (stability involving product of powers of norms) and generalized (Gavruta, 1994) (stability involving a general control function).

For the first time, the reciprocal functional equation

$$
\chi(u+v)=\frac{\chi(u) \chi(v)}{\chi(u)+\chi(v)}
$$

was introduced and its primary stabilities relevant to Ulam stability study were obtained in the setting of non-zero real numbers (Ravi and Senthil Kumar, 2010a). The reciprocal function $\chi(u)=\frac{c}{u}$ is a solution of the functional equation (1.1). The functional equation (1.1) is related with the reciprocal formula arising in an electric circuit with a couple of parallel resistors. (Ravi et al., 2011) examined the basic stability results of the Reciprocal Difference Functional (RDF) equation and Reciprocal Adjoint Functional (RAF) equation. In recent times, (Ravi and Suresh, 2017) the generalized Hyers-Ulam stability of reciprocal-quadratic functional equation in two variables has been investigated in the form

$$
R_{q}(u+v)=\frac{R_{q}(u) R_{q}(v)}{R_{q}(u)+R_{q}(v)+2 \sqrt{R_{q}(u) R_{q}(v)}}
$$

in the setting of non-zero real numbers. It is easy to verify that the reciprocal-quadratic function $R_{q}(u)=\frac{k}{u^{2}}$ is a solution of equation (1.2). There are many significant and remarkable stability results of various forms of reciprocal functional equations are (Kim et al., 2017), (Ravi et al., 2010b), (Ravi et al.,

*Authorfor Correspondence: E-mail:hemen_dutta08@rediffmail.com

\#Present address: Section of Mathematics, Department of Information Technology, Nizwa College of Technology, Nizwa 611, Oman 
2010c), (Ravi and Senthil Kumar, 2012), (Senthil Kumar and Bodaghi, 2017), (Senthil Kumar and Bodaghi, 2020), (Senthil Kumar et al., 2016a), (Senthil Kumar and Ravi, 2016), (Senthil Kumar et al., 2016b).

We also elicit here, a few primitive ideas of quasi- $\beta$-normed spaces. Let $\beta$ be a fixed real number with $0<\beta \leq 1$ and let $\mathbb{K}$ denote either $\mathbb{R}$ or $\mathbb{C}$.

Definition 1.1 - Let $\mathcal{X}$ be a linear space over $\mathbb{K}$. A quasi- $\beta$-norm is a real-valued function on $\mathcal{X}$ satisfying the following conditions: (i) $\|u\| \geq 0$, $\forall u \in \mathcal{X}$ and $\|u\|=0$ if and only if $u=0$; (ii) $\|\mu u\|=|u|^{\beta} .\|u\|, \forall \mu \in \mathbb{K}$ and $\forall u \in \mathcal{X}$; (iii) there is a constant $K>1$ such that $\|u+v\|<K(\|u\|+\|v\|)$, $\forall u, v \in \mathcal{X}$

The pair $(\chi,\|\|$.$) is called quasi- \beta$-normed space if $\|$.$\| is a quasi- \beta$-norm on $\chi$. The smallest possible $K$ is called the modulus of concavity of $\|$.$\| .$

Definition 1.2 - A quasi- $\beta$-Banach space is a complete quasi- $\beta$-normed space.

In this work, we are interested to ascertain geometrical meaning of the following ReciprocalQuadratic Difference (RQD) functional equation in several variables

$$
\begin{aligned}
& r\left(\frac{1}{n} \sum_{i=1}^{n} y_{i}\right)-r\left(\sum_{i=1}^{n} y_{i}\right) \\
& =\frac{\left(n^{2}+1\right) \prod_{i=1}^{n} r\left(y_{i}\right)}{\left[\sqrt{\sum_{i=1}^{n} r\left(y_{i}\right) \prod_{j=1 j \neq i}^{n} r\left(y_{j}\right)}\right]^{2}}
\end{aligned}
$$

and Reciprocal-Quadratic Adjoint (RQA) functional equation in several variables

$$
\begin{aligned}
r\left(\frac{1}{n} \sum_{i=1}^{n} y_{i}\right) & +r\left(\sum_{i=1}^{n} y_{i}\right) \\
& =\frac{\left(n^{2}+1\right) \prod_{i=1}^{n} r\left(y_{i}\right)}{\left[\sqrt{\sum_{i=1}^{n} r\left(y_{i}\right) \prod_{j=1 j \neq i}^{n} r\left(y_{j}\right)}\right]^{2}}
\end{aligned}
$$

and to investigate various fundamental stabilities in the framework of quasi- $\beta$-normed spaces.

Remark 1.3 : When $n=3$ in the above equations, we have

$$
\begin{aligned}
& r\left(\frac{y_{1}+y_{2}+y_{3}}{3}\right)-r\left(y_{1}+y_{2}+y_{3}\right)= \\
& \frac{8 r\left(y_{1}\right) r\left(y_{2}\right) r\left(y_{3}\right)}{\left[\sqrt{r\left(y_{1}\right) r\left(y_{2}\right)}+\sqrt{r\left(y_{2}\right) r\left(y_{3}\right)}+\sqrt{r\left(y_{1}\right) r\left(y_{3}\right)}\right]^{2}}
\end{aligned}
$$

and

$$
\begin{aligned}
& r\left(\frac{y_{1}+y_{2}+y_{3}}{3}\right)+r\left(y_{1}+y_{2}+y_{3}\right)= \\
& \frac{10 r\left(y_{1}\right) r\left(y_{2}\right) r\left(y_{3}\right)}{\left[\sqrt{r\left(y_{1}\right) r\left(y_{2}\right)}+\sqrt{r\left(y_{2}\right) r\left(y_{3}\right)}+\sqrt{r\left(y_{1}\right) r\left(y_{3}\right)}\right]^{2}}
\end{aligned}
$$

Throughout this paper, let $\mathcal{X}$ be a quasi- $\beta$ normed space and let $\mathcal{Y}$ be a quasi- $\beta$-Banach space with a quasi- $\beta$-norm $\|\cdot\|_{2}$. For a given mapping $r: \mathcal{X}$ $\rightarrow \mathcal{Y}$, let us define the difference operators

$$
\begin{aligned}
& D_{1} r, D_{2} r: \underbrace{\mathcal{X} \times \mathcal{X} \times \ldots \times \mathcal{X}}_{\text {ntimes }} \rightarrow \mathcal{Y} \text { by } \\
& D_{1} r\left(y_{1}, y_{2}, \ldots, y_{n}\right)=r\left(\frac{1}{n} \sum_{i=1}^{n} y_{i}\right) \\
& \quad-r\left(\sum_{i=1}^{n} y_{i}\right)-\frac{\left(n^{2}-1\right) \prod_{i=1}^{n} r\left(y_{i}\right)}{\left[\sqrt{\sum_{i=1}^{n} r\left(y_{i}\right) \prod_{j=1, j \neq i}^{n} r\left(y_{j}\right)}\right]^{2}}
\end{aligned}
$$

and

$$
\begin{aligned}
D_{1} r\left(y_{1}, y_{2}, \ldots, y_{n}\right) & =r\left(\frac{1}{n} \sum_{i=1}^{n} y_{i}\right) \\
& +r\left(\sum_{i=1}^{n} y_{i}\right)-\frac{\left(n^{2}-1\right) \prod_{i=1}^{n} r\left(y_{i}\right)}{\left[\sqrt{\sum_{i=1}^{n} r\left(y_{i}\right) \prod_{j=1, j \neq i}^{n} r\left(y_{j}\right)}\right]^{2}}
\end{aligned}
$$

for all $y_{1}, y_{2}, \ldots, y_{n} \in \mathcal{X}$. 
We also assume that $\sum_{i=1}^{n} y_{i} \neq 0$, for all $y_{i} \in \mathcal{X}, i=1,2, \ldots, n$ and $r(y) \neq 0$, for all $y \in \mathcal{X}$.

\section{Geometrical Interpretations of Equations (1.3) and (1.4)}

In this section, we establish the geometrical descriptions of functional equations (1.3) and (1.4) when $n=2$ using Newton's law of gravitation.

Newton's law of universal gravitation states that a particle attracts every other particle in the universe with a force which is directly proportional to the product of their masses and inversely proportional to the square of the distance between their centres.

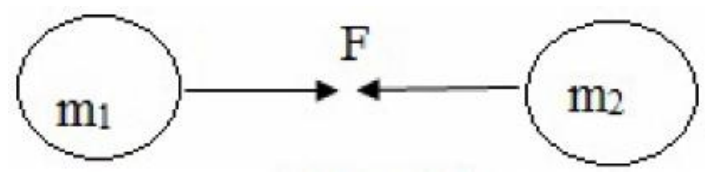

That is,

$$
F=G \frac{m_{1} m_{2}}{r^{2}}
$$

where $F$ is the force between the masses; $G$ is the gravitational constant; $m_{1}$ is the first mass; $m_{2}$ is the second mass and $r$ is the distance between the centres of the masses.

Suppose both the above two objects are of unit mass, then the force of attraction between them is

$$
F=\frac{G}{x^{2}}
$$

where $x$ is the distance between the objects.

Now, consider three objects of unit mass with the following situation. Let $x_{1}$ be the distance between object 1 and object 2 . Let $x_{2}$ be the distance between object 2 and object 3 .

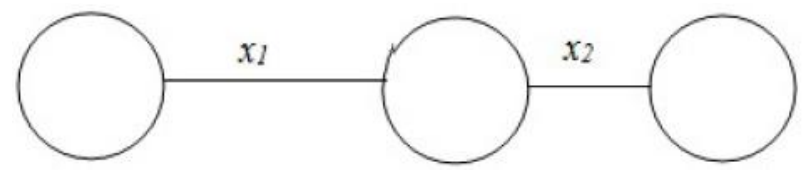

The force of attraction between object 1 and object 2 is

$$
F\left(x_{1}\right)=\frac{G}{x_{1}^{2}} .
$$

The force of attraction between object 2 and object 3 is

$$
F\left(x_{2}\right)=\frac{G}{x_{2}^{2}} .
$$

The force of attraction between object 1 and object 3 is

$$
F\left(x_{1}+x_{2}\right)=\frac{G}{\left(x_{1}+x_{2}\right)^{2}} .
$$

Using (1), (2) and (3), we have a relation as follows:

$$
\begin{aligned}
& F\left(x_{1}+x_{2}\right)=\frac{G}{x_{1}^{2}+x_{2}^{2}+2 x_{1} x_{2}} \\
&=\frac{G}{x_{1}^{2} x_{2}^{2}\left(\frac{1}{x_{1}^{2}}+\frac{1}{x_{2}^{2}}+\frac{2}{x_{1} x_{2}}\right)} \\
&=\frac{\frac{G}{x_{1}^{2} x_{2}^{2}}}{\frac{1}{x_{1}^{2}}+\frac{2}{x_{2}^{2}}+\frac{2}{x_{1} x_{2}}} \\
&=\frac{F\left(x_{1}\right) F\left(x_{2}\right)}{F\left(x_{1}\right)+F\left(x_{2}\right)+2 \sqrt{F\left(x_{1}\right) F\left(x_{2}\right)}}
\end{aligned}
$$

It is not hard to verify that the reciprocalquadratic function $F(x)=\frac{c}{x^{2}}$, where $c$ is a constant, is a solution of the above equation (2.4). Thus the reciprocal-quadratic functional equation (2.4) arises in the above physical phenomena.

If the distance between object 1 and object 3 is halved, that is, $\frac{x_{1}+x_{2}}{2}$, then the force of attraction between them becomes, 


$$
F\left(\frac{x_{1}+x_{2}}{2}\right)=\frac{G}{\left(\frac{x_{1}+x_{2}}{2}\right)^{2}}=4 \frac{G}{\left(x_{1}+x_{2}\right)^{2}}
$$

The relation between the difference of the forces $F\left(\frac{x_{1}+x_{2}}{2}\right)$ and $F\left(x_{1}+x_{2}\right)$ and the forces between object 1 and object 2; and object 2 and object 3 can be modeled as a functional equation as follows:

$$
\begin{gathered}
F\left(\frac{x_{1}+x_{2}}{2}\right)-F\left(x_{1}+x_{2}\right)=\frac{3 G}{\left(x_{1}+x_{2}\right)^{2}} \\
=\frac{3 F\left(x_{1}\right) F\left(x_{2}\right)}{F\left(x_{1}\right)+F\left(x_{2}\right)+2 \sqrt{F\left(x_{1}\right) F\left(x_{2}\right)}} .
\end{gathered}
$$

We call the above functional equation (2.5) as Reciprocal-Quadratic Difference Functional Equation (RQDF Equation) in two variables $x_{1}$ and $x_{2}$.

Similarly, the relation between the sum of the forces $F\left(\frac{x_{1}+x_{2}}{2}\right)$ and $F\left(x_{1}+x_{2}\right)$ and the forces between object 1 and object 2; and object 2 and object 3 can be modeled as a functional equation as follows:

$$
\begin{gathered}
F\left(\frac{x_{1}+x_{2}}{2}\right)+F\left(x_{1}+x_{2}\right)=\frac{5 G}{\left(x_{1}+x_{2}\right)^{2}} \\
=\frac{5 F\left(x_{1}\right) F\left(x_{2}\right)}{F\left(x_{1}\right)+F\left(x_{2}\right)+2 \sqrt{F\left(x_{1}\right) F\left(x_{2}\right)}} .
\end{gathered}
$$

The above functional equation (2.6) is said to be Reciprocal-Quadratic Adjoint Functional Equation (RQAF Equation) with a couple of variables $x_{1}$ and $x_{2}$.

\section{Fundamental Stabilities of Equations (1.3) and (1.4)}

In this section, we carry out the fundamental stability pertinent to Gavruta and then perpetuate to the stability results of the functional equations (1.3) and (1.4) in quasi- $\beta$-normed spaces pertinent to (Hyers, 1941), (Rassias, 1978) and (Rassias, 1982).
Theorem 3.1 - Assume $\psi: \underbrace{\mathcal{X} \times \mathcal{X} \times \ldots \mathcal{X}}_{n \text { times }}$ $\rightarrow[0, \infty)$ is a mapping fulfilling the following inequality:

$$
\sum_{j=0}^{\infty}\left(\frac{K}{n^{2 \beta}}\right)^{j} \psi\left(\frac{y_{1}}{n^{j+1}}, \frac{y_{2}}{n^{j+1}}, \ldots, \frac{y_{n}}{n^{j+1}}\right)<\infty
$$

for all $y_{1}, y_{2} \ldots, y_{n} \in \mathcal{X}$. Suppose $r: \mathcal{X} \rightarrow \mathcal{Y}$ is a mapping which gratifies the ensuing inequality

$$
\left\|D_{1} r\left(y_{1}, y_{2}, \ldots, y_{n}\right)\right\|_{\mathcal{Y}} \leq \psi\left(y_{1}, y_{2}, \ldots, y_{n}\right)
$$

for all $y_{1}, y_{2}, \ldots, y_{n} \in \mathcal{X}$. Then we can construct a unique reciprocal-quadratic mapping $R_{d}: \mathcal{X} \rightarrow \mathcal{Y}$ satisfying (1.3) and

$$
\begin{aligned}
\| r(y) & -R_{d}(y) \|_{\mathcal{Y}} \\
& \leq K \sum_{j=0}^{\infty}\left(\frac{K}{n^{2 \beta}}\right)^{j} \psi\left(\frac{y}{n^{j+1}}, \frac{y}{n^{j+1}}, \ldots, \frac{y}{n^{j+1}}\right)
\end{aligned}
$$

for all $y \in \chi$. The mapping $R_{d}(y)$ can be described as

$$
R_{d}(y)=\lim _{m \rightarrow \infty} \frac{1}{n^{2 m}} r\left(\frac{y}{n^{m}}\right) \text {, for all } y \in \chi .
$$

Proof : Switching $\left(y_{1}, y_{2}, \ldots, y_{n}\right)$ to $\left(\frac{y}{n}, \frac{y}{n}, \ldots, \frac{y}{n}\right)$ in (3.2) and simplifying further, we obtain

$$
\left\|r(y)-\frac{1}{n^{2}} r\left(\frac{y}{n}\right)\right\|_{\mathcal{Y}} \leq \psi\left(\frac{y}{n}, \frac{y}{n}, \ldots, \frac{y}{n}\right)
$$

for all $y \in \mathcal{X}$. Now, replacing $y$ by $\frac{x}{n}$, dividing by $n^{2 \beta}$ in (3.4) and summing the resulting inequality with (3.4), we find

$$
\begin{gathered}
\left\|r(y)-\frac{1}{n^{4}} r\left(\frac{y}{n^{2}}\right)\right\|_{\mathcal{Y}} \leq K \sum_{j=1}^{1}\left(\frac{K}{n^{2 \beta}}\right)^{j} \\
\psi\left(\frac{y}{n^{j+1}}, \frac{y}{n^{j+1}}, \ldots, \frac{y}{n^{j+1}}\right)
\end{gathered}
$$


for all $y \in \chi$. Proceeding further in the similar fashion and applying induction arguments on an integer $m>$ 0 , we arrive

$$
\begin{aligned}
\| r(y)- & \frac{1}{n^{2 m}} r\left(\frac{y}{n^{m}}\right) \|_{\mathcal{Y}} \\
& \leq K \sum_{j=1}^{m-1}\left(\frac{K}{n^{2 \beta}}\right)^{j} \psi\left(\frac{y}{n^{j+1}}, \frac{y}{n^{j+1}}, \ldots, \frac{y}{n^{j+1}}\right)
\end{aligned}
$$

for all $y \in \chi$. From (3.4), we obtain

$$
\begin{aligned}
\| \frac{1}{n^{2 j}} r\left(\frac{y}{n^{j}}\right) & -\frac{1}{n^{2(j+1)}} r\left(\frac{y}{n^{j+1}}\right)_{\mathcal{Y}} \| \\
& \leq \frac{1}{n^{2 j \beta}} \psi\left(\frac{y}{n^{j+1}}, \frac{y}{n^{j+1}}, \ldots, \frac{y}{n^{j+1}}\right)
\end{aligned}
$$

for all $y \in \chi$. For $m>1$,

$$
\begin{aligned}
\| \frac{1}{n^{2 m}} & r\left(\frac{y}{n^{m}}\right)-\frac{1}{n^{2 l}} r\left(\frac{y}{n^{l}}\right) \|_{\mathcal{Y}} \\
& \leq \sum_{j=l}^{m-1} \frac{1}{n^{2(j+1)}}\left\|r\left(\frac{y}{n^{j+1}}\right)-\frac{1}{n^{2 j}} r\left(\frac{y}{n^{j}}\right)\right\|_{\mathcal{Y}} \\
& \leq \sum_{j=l}^{m-1} \frac{1}{n^{2 j \beta}} \Psi\left(\frac{y}{n^{j+1}}, \frac{y}{n^{j+1}}, \ldots, \frac{y}{n^{j+1}}\right)
\end{aligned}
$$

for all $y \in \chi$. It is easy to find that the right-hand side of the above inequality (3.7) approaches to 0 as $m \rightarrow \infty$ which implies that the sequence $\left\{\frac{1}{n^{2 m}} r\left(\frac{y}{n^{m}}\right)\right\}$ is a Cauchy sequence in $\mathcal{Y}$. Hence, we can delineate

$$
R_{d}(y)=\lim _{m \rightarrow \infty} \frac{1}{n^{2 m}} r\left(\frac{y}{n^{m}}\right)
$$

for all $y \in \chi$. In lieu of $K \geq 1$, switching $\left(y_{1}, y_{2}, \ldots, y_{n}\right)$ to $\left(\frac{y_{1}}{n^{m}}, \frac{y_{2}}{n^{m}}, \ldots, \frac{y_{n}}{n^{m}}\right)$ and then dividing by $n^{2 m \beta}$ in (3.2), we have

$$
\begin{aligned}
\frac{1}{n^{2 m \beta}} & \left\|D_{1} r\left(\frac{y_{1}}{n^{m}}, \frac{y_{2}}{n^{m}}, \ldots, \frac{y_{n}}{n^{m}}\right)\right\|_{\mathcal{Y}} \\
& \leq \frac{K^{m}}{n^{2 m \beta}} \Psi\left(\frac{y_{1}}{n^{m}}, \frac{y_{2}}{n^{m}}, \ldots, \frac{y_{n}}{n^{m}}\right)
\end{aligned}
$$

for all $y_{1}, y_{2}, \ldots, y_{n} \in \chi$. Allowing $m \rightarrow \infty$ in (3.8) and using the definition of $R_{d}$, we observe that $R_{d}$ satisfies (3.3) for all $y_{1}, y_{2}, \ldots, y_{n} \in \mathcal{X}$. Hence $R_{d}$ is a reciprocal-quadratic mapping. Also, the inequality (3.5) produces the inequality (3.3). Next is to show the uniqueness of $\mathcal{X} \rightarrow \mathcal{Y}$. Presume that there subsists $R_{d}: \mathcal{X} \rightarrow \mathcal{Y}$ gratifying (1.3) and (3.3). It is easy to show that for all $y \in \mathcal{X}, R_{d}^{\prime}\left(\frac{y}{n^{m}}\right)=n^{2 m} R_{d}^{\prime}(y)$ and $R_{d}\left(\frac{y}{n^{m}}\right)=n^{2 m} R_{d}(y)$. Then

$$
\begin{aligned}
& \left\|R_{d}^{\prime}(y)-R_{d}(y)\right\|_{\mathcal{Y}}=\left\|\frac{1}{n^{2 m}} R_{d}^{\prime}\left(\frac{y}{n^{m}}\right)-\frac{1}{n^{2 m}} R_{d}\left(\frac{y}{n^{m}}\right)\right\|_{\mathcal{Y}} \\
& \leq \frac{K}{n^{2 m \beta}}\left(\left\|R_{d}^{\prime}\left(\frac{y}{n^{m}}\right)-r\left(\frac{y}{n^{m}}\right)\right\|_{\mathcal{Y}}+\left\|r\left(\frac{y}{n^{m}}\right)-R_{d}\left(\frac{y}{n^{m}}\right)\right\|_{\mathcal{Y}}\right) \\
& \leq 2 K^{2} \sum_{j=0}^{\infty}\left(\frac{K}{n^{2 \beta}}\right)^{m+j} \psi\left(\frac{y}{n^{m+j+1}}, \frac{y}{n^{m+j+1}}, \ldots, \frac{y}{n^{m+j+1}}\right)
\end{aligned}
$$

for all $y \in \mathcal{X}$. By letting $m \rightarrow \infty$, we immediately have the uniqueness of $R_{d}$.

The subsequent theorem is dual of Theorem 3.1. Hence, we present the main part of the proof as it is analogous to Theorem 3.1 .

$$
\text { Theorem 3.2 - Let } \psi: \underbrace{\mathcal{X} \times \mathcal{X} \times \ldots \times \mathcal{X}}_{n \text { times }}
$$
$\rightarrow[0, \infty)$ be a mapping gratifying the subsequent inequality:

$$
\sum_{n=0}^{\infty}\left(K n^{2 \beta}\right)^{j} \psi\left(n^{j} y_{1}, n^{j} y_{2}, \ldots, n^{j} y_{n}\right)<\infty \text { (3.9) }
$$

for all $y_{1}, y_{2}, \ldots, y_{n} \in \mathcal{X}$. Let $r: \mathcal{X} \rightarrow \mathcal{Y}$ be a mapping 
satisfying (3.2) for all $y_{1}, y_{2}, \ldots, y_{n} \in \mathcal{X}$. Then there subsists an inimitable reciprocal-quadratic mapping $R_{d}: \mathcal{X} \rightarrow \mathcal{Y}$ satisfying (1.3) and

$$
\begin{aligned}
& \left\|r(y)-R_{d}(y)\right\|_{\mathcal{Y}} \\
& \quad \leq n^{2 \beta} K \sum_{j=0}^{\infty}\left(n^{2 \beta} K\right)^{j} \psi\left(n^{j} y, n^{j} y, \ldots, n^{j} y\right)
\end{aligned}
$$

for all $y \in \chi$. The mapping $R_{d}(y)$ is defined by $R_{d}(y)=\lim _{m \rightarrow \infty} n^{2 m} r\left(n^{m} y\right)$, for all $y \in \chi$.

Proof: Switching $\left(y_{1}, y_{2}, \ldots, y_{n}\right)$ into $(y, y, \ldots, y)$ in (3.2) and multiplying by $n^{2 \beta}$, we obtain

$$
\left\|n^{2} r(n y)-r(y)\right\|_{\mathcal{Y}} \leq n^{2 \beta} \psi(y, y, \ldots, y)
$$

for all $y \in \chi$. Now, substituting $y$ as $n y$, multiplying by $n^{2 \beta}$ in (3.11) and summing the resulting inequality with (3.11), we have

$$
\begin{aligned}
& \left\|n^{4} r\left(n^{2} y\right)-r(x)\right\|_{\mathcal{Y}} \\
& \quad \leq n^{2 \beta} K \sum_{j=0}^{1}\left(n^{2 \beta} K\right)^{j} \psi\left(n^{j} y, n^{j} y, \ldots, n^{j} y\right)
\end{aligned}
$$

for all $y \in \chi$. Using induction arguments on a positive integer $m$, we conclude that

$$
\begin{aligned}
& \left\|n^{2 m} r\left(n^{m} y\right)-r(y)\right\|_{\mathcal{Y}} \\
& \quad \leq n^{2 \beta} K \sum_{j=0}^{m-1}\left(n^{2 \beta} K\right)^{j} \psi\left(n^{j} y, n^{j} y, \ldots, n^{j} y\right)
\end{aligned}
$$

for all $y \in \chi$. The rest of the proof is analogous to Theorem 3.1.

The following corollaries are immediate consequences of Theorems 3.1 and 3.2 related to the results of (Hyers, 1941), (Rassias, 1978) and (Rassias, 1982).

Corollary 3.3 - Let $\varepsilon \geq 0$ be fixed. If a mapping $r: x \rightarrow y$ satisfies the inequality $\left\|D_{1} r\left(y_{1}, y_{2}, \ldots, y_{n}\right)\right\|_{\mathcal{Y}} \leq \varepsilon$, for all $y_{1}, y_{2}, \ldots, y_{n} \in \mathcal{X}$, then there exists a unique reciprocal-quadratic mapping $R_{d}: \mathcal{X} \rightarrow \mathcal{Y}$ satisfying (1.3) amd
$\left\|r(y)-R_{d}(y)\right\|_{\mathcal{Y}} \leq \frac{n^{2 \beta} K \varepsilon}{n^{2 \beta}-K}$, for all $y \in \chi$.

Proof : Considering $\psi\left(y_{1}, y_{2}, \ldots, y_{n}\right)=\varepsilon$ in Theorem 3.1, we arrive at the desired result.

Corollary 3.4 - Let $\delta \geq 0$ be fixed and $\alpha \neq 2 \beta$. If a mapping $r: \mathcal{X} \rightarrow \mathcal{Y}$ satisfies the inequality $\left\|D_{1} r\left(y_{1}, y_{2}, \ldots, y_{n}\right)\right\|_{\mathcal{Y}} \leq \delta \sum_{i=1}^{n}\left\|y_{i}\right\|_{\mathcal{X}}^{\alpha}$, for all $y_{1}, y_{2}, \ldots, y_{n} \in \mathcal{X}$, then there exists a unique reciprocal-quadratic mapping $R_{d}: \mathcal{X} \rightarrow \mathcal{Y}$ satisfying (1.3) and

$\mid r(y)-R_{d}(y) \|_{\mathcal{Y}} \leq\left\{\begin{array}{l}\frac{n^{2 \beta+1} K \delta}{n^{2 \beta+\alpha}-K}\|y\|_{\chi}^{\alpha}, \text { for } \alpha>-2 \beta, \\ \frac{n^{2 \beta+1} K \delta}{1-n^{2 \beta+\alpha} K}\|y\|_{\chi}^{\alpha}, \text { for } \alpha<-2 \beta\end{array}\right.$

for all $y \in \chi$.

Proof : By choosing $\psi\left(y_{1}, y_{2}, \ldots, y_{n}\right)=$ $\delta \sum_{i=1}^{n}\left\|y_{i}\right\|_{\mathcal{X}}^{\alpha}$ and $\alpha>-2 \beta$ in Theorem 3.1 and $\alpha<-$ $2 \beta$ in Theorem 3.2, respectively, we obtain the requisite results.

Corollary 3.5 - Let $\delta \geq 0$ be a fixed real number and a be such that $\alpha \neq-2 \beta$. If $a$ mapping $R_{d}: \mathcal{X} \rightarrow \mathcal{Y}$ satisfies the inequality $\left\|D_{1} r\left(y_{1}, y_{2}, \ldots, y_{n}\right)\right\|_{\mathcal{Y}} \leq \delta \prod_{i=1}^{n} y_{i}^{\frac{\alpha}{n}}$ for all $y_{1}, y_{2}, \ldots$, $y_{n} \in \mathcal{X}$, then there exists a unique reciprocalquadratic mapping $R_{d}: \mathcal{X} \rightarrow \mathcal{Y}$ satisfying (1.3) and

$$
\left\|r(y)-R_{d}(y)\right\|_{\mathcal{Y}} \leq\left\{\begin{array}{l}
\frac{n^{2 \beta} K \delta}{n^{2 \beta+\alpha}-K}\|y\|_{\chi}^{\alpha}, \text { for } \alpha>-2 \beta, \\
\frac{n^{2 \beta} K \delta}{1-n^{2 \beta+\alpha} K}\|y\|_{\chi}^{\alpha}, \text { for } \alpha<-2 \beta
\end{array}\right.
$$

for all $y \in \chi$. 
Proof : By replacing $\psi\left(y_{1}, y_{2}, \ldots, y_{n}\right)=$ $\delta \prod_{i=1}^{n}\left\|y_{i}\right\|^{\alpha / n}$ and considering $\alpha>-2 \beta$ in Theorem

3.1 and $\alpha<-2 \beta$ in Theorem 3.2, we acquire required results.

The following theorems and corollaries are the various stability results investigated for the reciprocalquadratic adjoint functional equation (1.4). Since arguments of proofs are akin to the above theorems and corollaries, we present the statements only.

$$
\text { Theorem 3.6 Let } \psi: \underbrace{\mathcal{X} \times \mathcal{X} \times \ldots \mathcal{X}}_{n \text { times }} \rightarrow[0, \infty)
$$
be a mapping gratifying the following inequality:

$$
\sum_{j=0}^{\infty}\left(\frac{K}{n^{2 \beta}}\right)^{j} \psi\left(\frac{y_{1}}{n^{j+1}}, \frac{y_{2}}{n^{j+1}}, \ldots, \frac{y_{n}}{n^{j+1}}\right)<\infty
$$

for all $y_{1}, y_{2}, \ldots, y_{n} \quad \mathcal{X}$. Let $r: \mathcal{X} \rightarrow \mathcal{Y}$ be a mapping such that

$$
\left\|D_{2} r\left(y_{1}, y_{2}, \ldots, y_{n}\right)\right\|_{\mathcal{Y}} \leq \psi\left(y_{1}, y_{2}, \ldots, y_{n}\right)
$$

for all $y_{1}, y_{2}, \ldots, y_{n} \in \mathcal{X}$. Then there persists a distinctive reciprocal-quadratic mapping $R_{a}: \mathcal{X} \rightarrow \mathcal{Y}$ satisfying (1.4) and

$$
\begin{gathered}
\left\|r(y)-R_{a}(y)\right\|_{Y} \leq K \sum_{j=0}^{\infty}\left(\frac{K}{n^{2 \beta}}\right)^{j} \\
\psi\left(\frac{y}{n^{j+1}}, \frac{y}{n^{j+1}}, \ldots, \frac{y}{n^{j+1}}\right)
\end{gathered}
$$

for all $y \in \mathcal{X}$. The mapping $R_{d}(y)$ is defined by $R_{a}(y)=\lim _{m \rightarrow \infty} \frac{1}{n^{2 m}} r\left(\frac{y}{n^{m}}\right)$ for all $y \in \mathcal{X}$.

Theorem 3.7 - Let $\psi: \underbrace{\mathcal{X} \times \mathcal{X} \times \ldots \times \mathcal{X}}_{n \text { times }}$ $\rightarrow[0, \infty)$ be a mapping satisfying

$$
\sum_{j=0}^{\infty}\left(K n^{2 \beta}\right)^{j} \psi\left(n^{j} y_{1}, n^{j} y_{2}, \ldots, n^{j} y_{n}\right)<\infty
$$

for all $y_{1}, y_{2}, \ldots, y_{n} \in \mathcal{X}$. Let $r: \mathcal{X} \rightarrow \mathcal{Y}$ be a mapping satisfying (3.13) for all $y_{1}, y_{2}, \ldots, y_{n} \in \mathcal{X}$. Then there exists a unique reciprocal-quadratic mapping $R_{a}: \mathcal{X} \rightarrow \mathcal{Y}$ satisfying (1.4) and

$$
\begin{aligned}
& \left\|r(y)-R_{a}(y)\right\|_{\mathcal{Y}} \\
& \quad \leq n^{2 \beta} K \sum_{j=0}^{\infty}\left(n^{2 \beta} K\right)^{j} \psi\left(n^{j} y, n^{j} y, \ldots, n^{j} y\right)
\end{aligned}
$$

for all $y \in \mathcal{X}$. The mapping $R_{d}(y)$ is defined by $R_{a}(y)=\lim _{m \rightarrow \infty} n^{2 m} r\left(n^{m} y\right)$ for all $y \in \mathcal{X}$.

Corollary 3.8 - Let $\varepsilon \geqq 0$ be fixed. If a mapping $r: \mathcal{X} \rightarrow \mathcal{Y}$ satisfies the inequality $\left\|D_{2} r\left(y_{1}, y_{2}, \ldots, y_{n}\right)\right\|_{\mathcal{Y}} \leq \varepsilon$, for all $y_{1}, y_{2}, \ldots, y_{n} \in \mathcal{X}$, then there exists a unique reciprocal-quadratic mapping $R_{a}: \mathcal{X} \rightarrow \mathcal{Y}$ satisfying (1.4) amd $\left\|r(y)-R_{a}(y)\right\|_{\mathcal{Y}} \leq \frac{n^{2 \beta} K \varepsilon}{n^{2 \beta}-K}$, for all $y \in \mathcal{X}$.

Corollary 3.9 - Let $\delta \geq 0$ be fixed and $\alpha \neq 2 \beta$. If a mapping $r: \mathcal{X} \rightarrow \mathcal{Y}$ satisfies the inequality $\left\|D_{2} r\left(y_{1}, y_{2}, \ldots, y_{n}\right)\right\|_{\mathcal{Y}} \leq \delta \sum_{i=1}^{n}\left\|y_{i}\right\|_{\mathcal{X}}^{\alpha}$, for all $y_{1}, y_{2}, \ldots, y_{n} \in \mathcal{X}$, then there exists a unique reciprocal-quadratic mapping $R_{a}: \mathcal{X} \rightarrow \mathcal{Y}$ satisfying (1.4) and

$\left\|r(y)-R_{a}(y)\right\|_{\mathcal{Y}} \leq\left\{\begin{array}{l}\frac{n^{2 \beta+1} K \delta}{n^{2 \beta+\alpha}-K}\|y\|_{\chi}^{\alpha}, \text { for } \alpha>-2 \beta, \\ \frac{n^{2 \beta+1} K \delta}{1-n^{2 \beta+\alpha} K}\|y\|_{\chi}^{\alpha}, \text { for } \alpha<-2 \beta\end{array}\right.$

for all $y \in \mathcal{X}$.

Corollary 3.10 - Let $\delta \geq 0$ be a fixed real number and a be such that $\alpha \neq-2 \beta$. If a mapping $R_{a}: \mathcal{X} \rightarrow \mathcal{Y}$ satisfies the inequality $\left\|D_{1} r\left(y_{1}, y_{2}, \ldots, y_{n}\right)\right\|_{\mathcal{Y}} \leq \delta \prod_{i=1}^{n} y_{i}{ }^{\frac{\alpha}{n}} \quad$ for $\quad$ all 
$y_{1}, y_{2}, \ldots, y_{n} \in \mathcal{X}$, then there exists a unique reciprocal-quadratic mapping $R_{d}: \mathcal{X} \rightarrow \mathcal{Y}$ satisfying (1.4) and

$\left\|r(y)-R_{a}(y)\right\|_{\mathcal{Y}} \leq\left\{\begin{array}{l}\frac{n^{2 \beta} K \delta}{n^{2 \beta+\alpha}-K}\|y\|_{\chi}^{\alpha}, \text { for } \alpha>-2 \beta, \\ \frac{n^{2 \beta} K \delta}{1-n^{2 \beta+\alpha} K}\|y\|_{\chi}^{\alpha}, \text { for } \alpha<-2 \beta\end{array}\right.$

for all $y \in \mathcal{X}$.

\section{Comparison of The Results of Equations (1.3)} and (1.4) With the Existing Literture

In this section, we compare the results of equations (1.3) and (1.4) with the existing results available in the literature.

The Hyers-Ulam stability of reciprocal difference and adjoint functional equations involving two variables with their solutions as multiplicative inverse function $(x)=\frac{k}{x}$, were established in the setting of non-zero real numbers by substantiating an inexact solution is close to its correct solution (Ravi et al., 2010c).

In proving Hyers-Ulam stability results of the generalized reciprocal type functional equation comprising two variables with solution as $f(x)=\frac{k}{x}$, it has been proved that there exists an approximate solution near to the precise solution in the setting of non-Archimedean fields (Ravi et al., 2015).

The Hyers-Ulam stability results of reciprocalcubic and reciprocal-quartic functional equations containing two variables with solutions multiplicative inverse cubic and quartic functions, respectively, $c(x)=\frac{k}{x^{3}}$ and $q(x)=\frac{k}{x^{4}}$, were achieved by showing inexact solutions subsist near to their actual solutions in the framework of non-Archimedean fields et al., 2017).

The Hyers-Ulam stability of duodecic functional equation with solution as duodecic function $f(x)=x^{12}$, was demonstrated by determining an approximate solution close to the exact solution in the structure of quasi- $\beta$-normed spaces (Rassias et al., 2017).

The Hyers-Ulam stability of a quadratic reciprocal functional equation containing two variables only with solution as multiplicative inverse quadratic

function $f(x)=\frac{c}{x^{2}}$, was proved in the setting of nonArchimedean fields, by validating an approximate solution exists close to the actual solution (Bodaghi and Ebrahimdoost, 2016).

In the above literature, we observe that the Hyers-Ulam stability results of rational functional equations were attained in non-Archimedean fields. Inspired by results, in this study, we have dealt new reciprocal-quadratic and adjoint functional equations in several variables with their solution as multiplicative inverse quadratic function and acquired that their Hyers-Ulam stability results are still sustainable by showing their approximate solutions exist near to their exact solutions in the setting of quasi- $\beta$-normed spaces, which is a different space not dealt so far to investigate Hyers-Ulam stability of rational functional equations. Also, this is thefirst attempt in the theory of functional equations with illustrations of geometric interpretations of rational functional equations in physics.

\section{Acknowledgement}

The authors would like to thank the referees for careful reading and giving good suggestions to improve the quality of the paper. 


\section{References}

Aoki T (1950) On the stability of the linear transformation in Banach spaces J Math Soc Japan 2 64-66

Bodaghi A and Ebrahimdoost Y (2016) On the stability of quadratic reciprocal functional equation in nonArchimedean fields Asian-Euro J Math 9 1-9

Gavruta P (1994) A generalization of the Hyers-Ulam-Rassias stability of approximately additive mapppings $J$ Math Anal Appl 184 431-436

Hyers D H (1941) On the stability of the linear functional equation Proc Nat Acad Sci USA 27 222-224

KimS O, Senthil Kumar B V and Bodaghi A (2017) Stability and non-stability of the reciprocal-cubic and reciprocal-quartic functional equations in non-Archimedean fields $A d v$ Difference Equ 77 1-12

Rassias J M (1982) On approximation of approximately linear mappings by linear mappings J Funct Anal 46 126-130

Rassias J M, Ravi K and Senthil Kumar B V (2017) A fixed point approach to Ulam-Hyers stability of duodecic functional equation in quasi-ß-normed spaces Tbilisi Math J 10 83101

Rassias T M (1978) On the stability of the linear mapping in Banach spaces Proc Amer Math Soc 72 297-300

Ravi K, Rassias J M and Senthil Kumar B V (2010a) Ulam stability of generalized reciprocal functional equation in several variables Int J App Math Stat 19 1-19

Ravi K, Rassias J M and Senthil Kumar B V (2010b) Solution and stability of 2-variable reciprocal functional equation Bulletin Math Anal Appl 2 84-92

Ravi K, Rassias JM and Senthil Kumar B V (2010c) A fixed point approach to the generalized Hyers-Ulam stability of reciprocal difference and adjoint functional equations Thai J Math 8 469-481
Ravi K, Rassias J M and Senthil Kumar B V (2011) Ulam stability of reciprocal difference and adjoint functional equations Aust J Math Anal Appl 8 1-18

Ravi K, Rassias J M and Senthil Kumar B V (2015) Ulam stability of a generalized reciprocal type functional equation in nonArchimedean fields Arab J Math 4 117-126

Ravi K and Senthil Kumar B V (2010) Ulam-Gavruta-Rassias stability of Rassias reciprocal functional equation Global J Appl Math and Math Sci 3 57-79

Ravi K and Senthil Kumar B V (2012) Stability and geometrical interpretation of reciprocal functional equation Asian $J$ Current Engg Math 1 300-304

Ravi K and Suresh S (2017) Solution and generalized HyersUlam stability of a reciprocal quadratic functional equation Int J Pure Appl Math 119 Art. No. AP2017-31-4927

Senthil Kumar B V and Bodaghi A (2017) Estimation of inexact reciprocal-quintic and reciprocal-sextic functional equations Mathematica 593-14

Senthil Kumar B V and Bodaghi A (2020) Approximation of Jensen type reciprocal functional equation using fixed point technique Boletim da Sociedade Paranaense de Matematica 38 125-132

Senthil Kumar B V, Ravi K and Rassias J M (2016a) Solution and generalized Ulam-Hyers stability of a reciprocal type functional equation in non-Archimedean fields World Scientific News 31 71-81

Senthil Kumar B V and Ravi K (2016) Ulam stability of a reciprocal functional equation in quasi-beta-normed spaces Global $J$ Pure Appl Math 12 125-128

Senthil Kumar B V, Rassias J M and Ravi K (2016b) Ulam stability of a bi-reciprocal functional equation in quasi- $\beta$-normed spaces Novi Sad J Math 46 1-11

Ulam S M (1964) Problems in Modern Mathematics, Chapter VI, Wiley-Interscience, New York. 